\title{
Primary Teeth Stains and Discoloration: A Review
}

\author{
Abdulfatah Alazmah ${ }^{1}$ \\ 1 Pediatric Dentistry Division, Department of Preventive Dental \\ Science, College of Dentistry, Prince Sattam University, Alkharj, \\ Saudi Arabia \\ Address for correspondence Abdulfatah Alazmah, BDS, MFDRCSI, \\ Department of Preventive Dental Science, College of Dentistry, Prince \\ Sattam University, Alkharj 11942, Saudi Arabia \\ J Child Sci 2021;11:e20-e27. \\ (e-mail: A.alazmah@psau.edu.sa).
}

\begin{abstract}
Keywords

- pediatric dentistry

- oral health

- child

- tooth discoloration

The current review assesses the literature concerning the etiology of primary teeth staining and discoloration. The appearance of the dentition is of concern to many children and their caregivers seeking dental treatment as the color of the teeth is of aesthetic importance. The correct diagnosis of the cause of the discoloration is important as, invariably, it has a profound effect on treatment outcomes. It would seem reasonable, therefore, that dental practitioners understand the etiology of tooth discoloration to make a diagnosis and enable appropriate treatment to be conducted. Knowledge of the etiology of tooth staining is of importance to the dental practitioner to explain the patient the exact nature of the condition. In some instances, the mechanism of staining affects the outcome of the treatment and influences the options the dentist will be able to offer. Many of these contributing factors are preventable if parents are educated, managed by professional interventions, or provide a multidisciplinary approach. In this review, we highlight the importance of children smiles and the difference between primary and permanent teeth. We discuss the different types of primary teeth staining and discoloration and whether it is intrinsic or extrinsic, as well as the different impacts of some conditions on primary teeth compared with permanent teeth.
\end{abstract}

\section{Introduction}

Understanding the different etiological factors of staining and discoloration in primary teeth will lead to better management approaches and quality of dental treatments, meeting children's rights and improving their quality of life. Dental treatment can make a positive impact on the psychological and social aspects of a child's life. ${ }^{1,2}$ For pediatric dentists, it is essential to provide parents with information regarding the preventive methods to avoid deciduous teeth discoloration. Increasing population knowledge about tooth discoloration etiology, especially systemic diseases that can cause discoloration, the importance of oral hygiene, pregnancy and infancy care, and preventing injury to deciduous teeth should be a priority. ${ }^{3}$

received

August 22, 2020

accepted after revision

November 21, 2020
DOI https://doi.org/

10.1055/s-0040-1722276.

ISSN 2474-5871.
Teeth color is influenced by a combination of presence of any extrinsic stains that may form on the tooth surface and their intrinsic color. ${ }^{4}$ Teeth discoloration is defined as the variation from normal color, and this phenomenon can significantly affect the child's personality. ${ }^{5}$ Color variations can be present in an individual tooth from the incisal edge to the darker cervical area around the gingiva. ${ }^{6}$ Among the millions of color varieties, $\sim 100,000$ can be found in natural human teeth. $^{7}$ Color is a combination of the psychophysiological response to the physical interaction of light energy with an object and the subjective experience of an individual observer. ${ }^{8}$ In 1981, Munsell described color using the following three terms: chroma, hue, and value. ${ }^{9}$ The value reflects the lightness of the color and the chroma is the brightness reflecting its degree of strength and intensity. The hue enables the

\section{(c) 2021. The Author(s).}

This is an open access article published by Thieme under the terms of the Creative Commons Attribution License, permitting unrestricted use, distribution, and reproduction so long as the original work is properly cited. (https://creativecommons.org/licenses/by/4.0/)

Georg Thieme Verlag KG, Rüdigerstraße 14, 70469 Stuttgart, Germany 
individual to differentiate between the different families of color. ${ }^{10}$ Within the same dentition, tooth color can vary between different types of teeth, where canines are usually darker in color than others. ${ }^{11}$ For the tooth to appear in its total color, a combination of light will be created and then it will be scattered and reflected by different layers of enamel and the dentin underneath. ${ }^{6}$ The perception of color is influenced by three different factors: the object, the observer, and the light source. ${ }^{12}$ Other factors to be considered are the time of day and the angle of the tooth. ${ }^{13}$ As a result, a tooth will exhibit different colors when viewed under different conditions due to the differences in wavelength of the light source. ${ }^{14}$ According to Kim and Simmer, A1, A2, and B1 are the most common color shades in primary dentition. ${ }^{15}$

Primary teeth develop earlier than permanent teeth. An accurate chronology of primary teeth calcification is clinically significant to dentists. ${ }^{16,17}$ All primary teeth develop throughout the embryonic stage, starting from around 6 weeks and undergoing five stages of the tooth life cycle, including the following: initiation, proliferation, histodifferentiation, morpho-differentiation, apposition, and calcification. It is common to have a defect that includes primary teeth discoloration and staining as a result of a disturbance to any stages. ${ }^{16,17}$ Multiple variations have been found when comparing primary and permanent teeth. These are important to understand, as it is essential to address the difference in response to a condition affecting them and how to deliver an evidence-based treatment. There is a difference in the enamel and dentin thickness between primary and permanent teeth, in addition to a difference toward the enamel rods. A study compared both dentitions' mineral content, the thickness of the enamel, and enamel rods, and concluded the following: the primary enamel structure showed a lower calcium and phosphorus level, less thickness, and higher numerical density of rods. ${ }^{18}$

Finn, Nelson, and Ash identified 12 differences between deciduous and permanent teeth; deciduous teeth are smaller than their successors in all dimensions, a thinner layer of enamel and dentin thickness is noted with a wider pulp chamber having the pulp horns (specially the mesial horn) closer to the occlusal surface when compared with permanent molars. In addition, the crown of primary teeth is wider mesiodistally and the roots are longer and narrower. ${ }^{19,20}$ In staining, some conditions commonly affect permanent teeth, such as molar incisor hypomineralization. ${ }^{5}$ In contrast, other conditions affect primary teeth more severely, such as dentinogenesis imperfecta (DGI). ${ }^{21}$

Nondental primary care providers can improve children's oral health. There is a growing international interest in the role of pediatricians in children's oral health, ${ }^{22}$ especially considering the high caries rate affecting children with primary teeth ( $\sim 33 \%$ of 5 years old). While recent findings demonstrate limited knowledge regarding oral health, pediatric awareness of the risk factors and etiology of dental conditions will lead to a timely referral system and costeffective dental management. A study of dental awareness among pediatric postgraduate physicians in the United Kingdom concluded that collaboration with dentists should be done to ensure that education programs include information about the oral health of children. ${ }^{22,23}$

Since the United Nation convention on children's rights in 1987, research has shifted toward increasing involving children in decision making, including in dental treatments. ${ }^{24}$ Meeting patients' aesthetics demands is proven to motivate patients to adhere to preventive measures and to keep their teeth healthy. ${ }^{3}$ In addition, the psychological concerns of children and their parents or caregivers have been reported. A study of 1,140 adolescents concluded that multiple dental disorders, including caries, tooth loss, and dental trauma affected their self-esteem. ${ }^{25}$ Another study asked 40 children affected by amelogenesis imperfecta (AI) about the single most important feature they wanted following their dental treatment; the results show that more than $60 \%$ of children hoped for color improvement in their teeth. Furthermore, most children started to notice a difference in their teeth color at around 6 years of age due to a defect. ${ }^{26}$

The purpose of this study is to review the literature on the etiology of primary teeth staining and discoloration as there has been little mention in literature. ${ }^{27}$

\section{Classification of Tooth Discoloration}

The crown of the tooth is composed of the following three layers: enamel, dentin, and pulp. Any disturbance that occurs within these layers may affect the appearance and color of the tooth, caused by the light transmission and reflection properties of these layers. Teeth discoloration is classified as intrinsic and extrinsic based on the stain location, where each can be further classified into the subcategories shown in -Fig. $\mathbf{1}^{14}$

\section{Intrinsic Discoloration Factors}

Intrinsic discoloration occurs due to a change in the structural composition or thickness of the dental hard tissues, both the enamel and dentin. This means that the chromogenic material becomes incorporated within the dental hard tissue: either during tooth development (pre-eruption), such as dental fluorosis, tetracyclic stain, inherited development defects of enamel and dentin without any systemic features and hematologic disorders, or during the post-eruptive phase of tooth development, such as pulpal necrosis. These stains can be localized on one or several teeth, through trauma, inadequate or improper endodontic treatment, and staining due to restoration (amalgam staining). ${ }^{28}$

The treatment of intrinsic tooth discoloration staining conditions will depend on the cause, severity of the condition, and age of the child. However, appropriate management should include establishing prevention, pain and sensitivity control, improve the aesthetic, and using different behavior management techniques. Both partial (resin composite and glass ionomers) and full coverage (preformed crown) are used to restore defected primary teeth. ${ }^{29}$

\section{Dental Fluorosis}

Dental fluorosis causes generalized intrinsic staining due to excessive exposure to fluoride in both prenatal and postnatal 


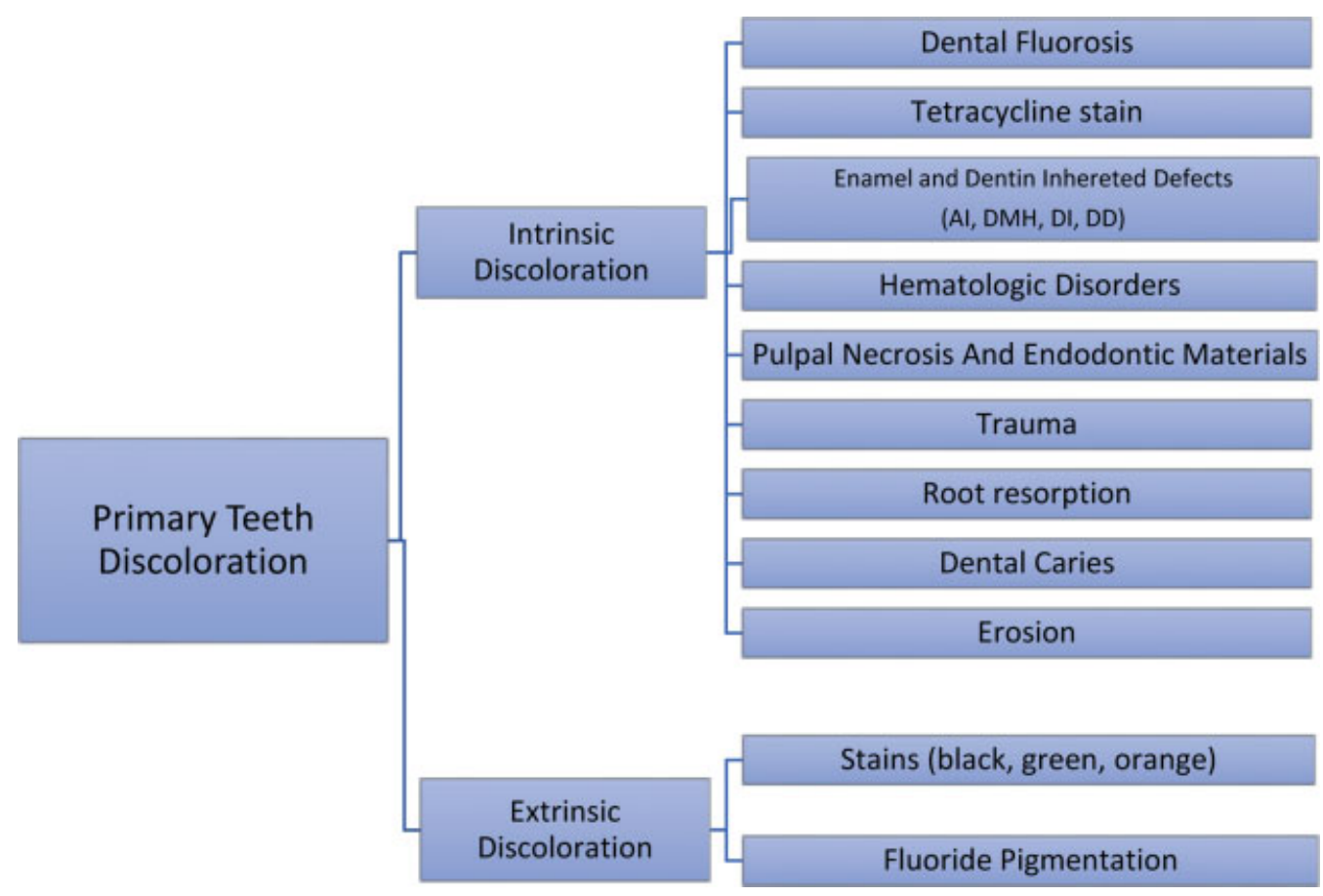

Fig. 1 Classification of teeth discoloration.

periods. It is the most common cause of intrinsic stain, especially in areas where drinking water has fluoride concentrations above optimal levels. Furthermore, the high prevalence may be due to the high availability of fluoride in multiple sources other than water. ${ }^{30,31}$

The severity differs based on the dose, duration of exposure, stage of ameloblastic activity, and variation in personal susceptibility. ${ }^{32,33}$ This excessive or chronic ingestion of fluoride during the enamel formation will cause hypomineralization or the porosity of this layer. In severe cases, the porosity will extend to the amelodentinal junction, which may lead to the breakdown of the enamel after tooth eruption. ${ }^{27}$ High concentrations of fluoride have an effect on the activity of the ameloblasts, with more clinical effect during the formation of the anterior teeth. ${ }^{34}$

The clinical appearance of dental fluorosis ranges from mild, gentle accentuation of perikymata pattern, to white opaque spots or streaks on the surface enamel, extending to brown pitting patches, to a more severe case of almost complete loss of the enamel layer. This phenomenon is less aggressive in primary teeth than permanent teeth and this can be attributed to shorter maturation and formation periods of primary teeth or the thinner enamel layer in them. ${ }^{27}$

\section{Tetracycline Stain}

Tetracycline stain was first reported in the mid-1950s. The Food and Drug Administration announced in 1963 the harm of such antibiotics to pregnant women and young children. ${ }^{35}$ The most critical time for tetracycline staining is during tooth formation (i.e., between the 2nd trimester intrauterine and 8 years of age). Deposition of tetracycline stain in the bone of the fetus will affect bone growth. If large amounts of tetracycline are consumed during the calcification stage, this will result in hypoplasia of dental hard tissues (i.e., enamel or dentin). ${ }^{36}$
The pattern of deposition occurs on the incremental lines of both dentin and enamel, giving the whole tooth a stained appearance. The mechanism of deposition is explained by the chelation action of the tetracycline molecule with the calcium present in the hydroxyapatite crystals that form a complex named tetracycline-calcium orthophosphate complex. ${ }^{36,37}$ Having a larger surface area of hydroxyapatite crystals, such in dentin, makes it susceptible to absorb a higher load of the tetracycline. After the administration of tetracycline, its chromogen is permanently present in the dental hard tissue; it can even be released throughout the normal bone remodeling. This constant release of tetracycline circulates freely in the bloodstream to reenter and be incorporated into other distant tissues undergoing calcification. ${ }^{38}$

The extent and severity of the stain depend on the time and duration of the drug's administration and the dosage used, affecting the extent, color, depth, and location of the stain ranging from light yellow to blue or brown in more severe cases. ${ }^{38}$ Minocycline, a tetracycline analog, may cause serious discoloration in teeth for both children and adults when taken for as short as 1 week. ${ }^{39}$

Diagnosis of the tetracycline stains is based on history, clinical presentation, and the fluorescence appearance under ultraviolet light (at $\sim 360 \mathrm{~nm}$ ). The labial surface of the anterior teeth is usually the first to get a darker color, whereas the more protected posterior teeth maintain a yellowish color for a longer period. ${ }^{40}$ When comparing the effect on both primary and permanent teeth, permanent teeth are less intense but more diffused compared with the primary teeth. ${ }^{41}$

\section{Inherited Development Defects of Enamel and Dentin Without Any Systemic Features}

$\mathrm{AI}$ is caused by gene mutation or altered expression. The term used when a group of hereditary conditions affects the tooth 
enamel appearance and structure, frequently associated with changes in other intraoral and/or extraoral tissues. The teeth are sensitive, fragile, and discolored, and are characterized by hypomineralization or hypoplasia. When a child's teeth are affected by AI, the clinical appearance and structure of all or the most of primary and secondary dentitions will be affected. The prevalence of AI differs depending on region or country of origin; however, the average global prevalence is less than 1 in $200 .{ }^{42}$ Aldred et al proposed a classification of AI based on different aspects that includes molecular basis, mode of inheritance, phenotype (appearance), and biochemical outcome. ${ }^{43}$ Depending on the appearance, the types of AI vary from the relatively mild hypomature "snow-capped" enamel to the more severe hereditary hypoplasia with thin, hard enamel, which has a yellow to yellow-brown appearance ${ }^{14}$ as shown in - Fig. 2 .

The etiology of $\mathrm{AI}$ is attributed to multiple fetal or maternal conditions. For example, a disturbance in the development of the tooth germ, such as deficiency of maternal vitamin D, infections (i.e., rubella), drug intake during pregnancy, and in pediatric hypocalcemia conditions. ${ }^{44}$ The time at which the interference in development is very important where it characterizes the degree of the systemic uptake.

Deciduous molar hypomineralization is another enamel defect leading to yellow-brown staining. The significance of this condition comes from the second primary molars develop in the same period of the first permanent molar. As a result, children with primary molars hypomineralization are at a greater risk of molar incisor hypomineralization; therefore, regular monitoring of these patients is recommended (-Fig. 3). ${ }^{45}$

Enamel hypoplasia in primary teeth has been widely reported in the literature. An association was found between enamel hypoplasia and premature birth, where it was the most frequently reported outcome. ${ }^{46}$ Opacity and hypoplasia are two outcomes of prematurity, referred to as "developmental enamel defects," where another study established a significant difference between exposed and nonexposed subjects. ${ }^{47}$

Heredity dentin disorder is a genetic group of autosomal dominant conditions including DGI and dentin dysplasia (DD). These conditions affect the dentin structures of primary teeth or both dentitions. DGI is more common than DD, the incidence is 1 in 6,000 to 8,000 and 1 in 100,000 , respectively. The defects cause teeth staining, which may vary from normal to amber, gray, or purple to bluish translucent discoloration. In addition to the discolored teeth

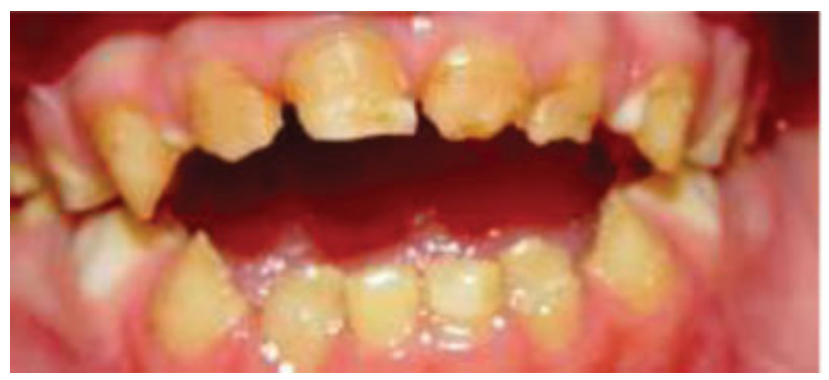

Fig. 2 Amelogenesis imperfecta.

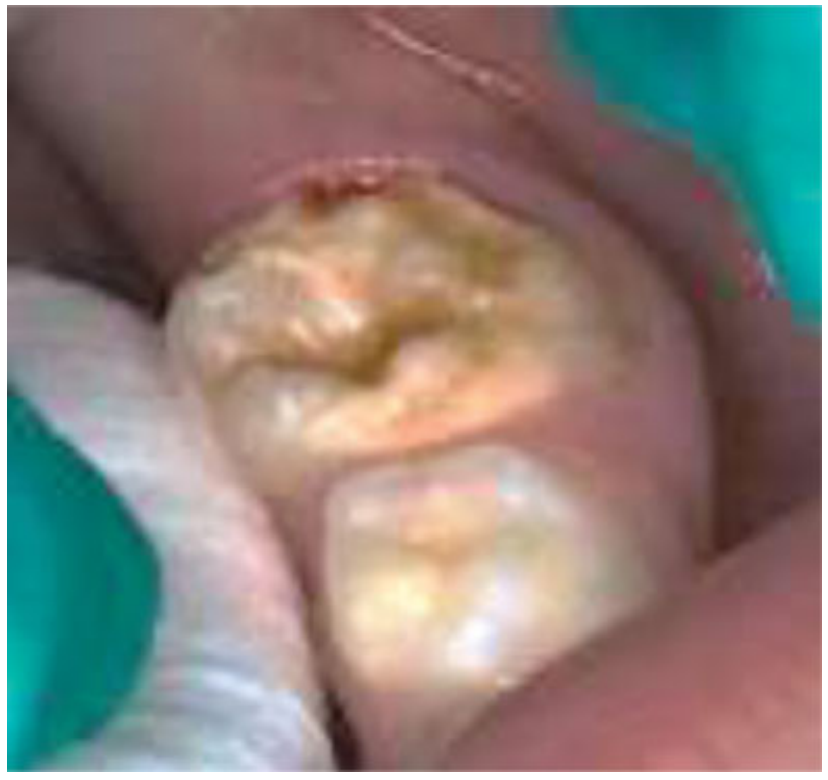

Fig. 3 Deciduous molar hypomineralization.

caused by these conditions, the teeth are translucent and often roughened with severe amber discoloration, structural defects such as a radiographically small pulp chamber and bulbous crowns. The defect in the mineralization process increases the potential of the enamel layer shearing that subsequently leads to wear in the dentinal layer. ${ }^{48}$

Shields classified DGI into three types and DD into two subcategories. ${ }^{49}$ DGI type I is the most common and the only type associated with genetic disorders that affect bones known as osteogenesis imperfecta. Radiographically, the roots of the teeth are short, constricted, and associated with dentin hypertrophy. Therefore, teeth experience pulp canal obliteration after or before their eruption. Clinically, it affects both dentitions with significant tooth surface loss. The color is translucent and typical amber. Other types include DGI type II, also called hereditary opalescent dentin, and the Brandywine isolate type III, found most commonly in Brandywine in the United States. ${ }^{50}$

\section{Hematologic Disorders}

Hematological disease, such as erythroblastosis fetalis and icterus gravis neonatorum, might produce jaundice in the newborn. This can cause a yellow-green discoloration and enamel hypoplasia in deciduous teeth. The reason for this yellow color is due to the deposition of bilirubin in the dental hard tissue during the developmental stage. Congenital erythropoietic porphyria is another disorder that causes purplish-red or reddish-brown discoloration to both deciduous and successor teeth. The discoloration is caused by the deposition of porphyrin pigments in bone and dentin. Diagnostically, the affected teeth can be detected with ultraviolet light under red fluorescence. ${ }^{27}$

\section{Trauma}

When children start to crawl, walk, and run, they are subject to unintentional falls and collisions that may result in trauma 
to the primary teeth, most commonly in children between 2 and 6 years of age. ${ }^{51}$ Some types of trauma will cause necrosis to the primary teeth. Keeping these teeth untreated will lead to resorption of both primary teeth and the supporting bone, this phenomenon will lead to early loss of primary teeth and early eruption of the successor. ${ }^{52}$

The color change of traumatized teeth is an early common sign witnessed by parents or guardians. ${ }^{53}$ Furthermore, discoloration is also considered an early sign of pulpal degeneration. ${ }^{54}$ When the pulp degenerates without hemorrhage, a stain of grayish-brown will appear caused by protein degeneration. ${ }^{55}$ The trauma causes a rupture of the blood vessels present in the pulp chamber, causing a breakdown of the blood and hemolysis of the erythrocytes that produce hemoglobin. Hemoglobin then gradually degrades to iron that shows as a bluish-black stain of the tooth, ${ }^{52,56}$ as shown in -Fig. 4.

Although it has been suggested that the gray discoloration of the crown is a sign of pulp necrosis, ${ }^{57}$ studies have shown that traumatized primary teeth might stay discolored (having a discolored gray crown) for several years with no other clinical or radiographic sign of pulpal degeneration or necrosis. ${ }^{54}$ Inadequate or improper endodontic treatment following trauma may lead to dark discoloration, which may occur due to incomplete removal of the pulpal tissue or incomplete removal of root canal filling material from the pulp chamber. ${ }^{27}$

\section{Amalgam Staining}

The demand for high aesthetics dental filling and the significant increase of minimal intervention of restorative approaches, considering the amalgam as a material of choice for primary teeth, has been reduced. ${ }^{58}$ The use of amalgam has been controversial due to concerns related to its mercury release. An in vivo study that included 20 primary molars indicated that amalgam causes bluish discoloration because of copper release. The grayish-black stain could be a result of an amalgam filling, which may occur due to the corrosion of amalgam, leading to the formation of silver sulfide. ${ }^{58,59}$

\section{Root Resorption}

The physiological pattern of root resorption of primary teeth occurs on the root surface, either within the pulp or from the

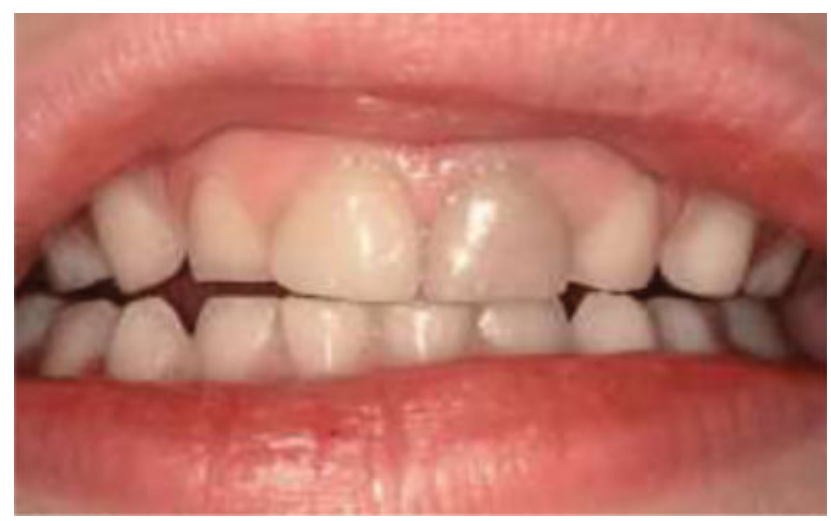

Fig. 4 Trauma for upper left primary central incisor. periodontal ligament (i.e., in the form of internal or external root resorption). The exact location of resorption is hard to detect until the lesion reaches a certain size. Clinically, it is asymptomatic, but occasionally it may appear as pink discoloration of the amelocemental junction. ${ }^{14,60}$

\section{Dental Caries}

Dental caries involves microorganisms excreting acid resulting in changes in dental tissue hardness and color. Dental caries is diagnosed by the change in color and may indicate the stage of the disease and lesion activity. The initial change in color due to caries is an opaque white spot as a result of increased enamel layer porosity due to acid production by the microbial metabolism of sugar. ${ }^{61}$ When caries develop further, a brown discolored dentin layer will be present, but the cause of discoloration remains controversial in the literature. ${ }^{62}$ However, particularly for primary teeth, the color and hardness of carious lesions are two clinical criteria used for the diagnosis of the progression degree of the disease. An active caries tends to be light yellow/beige, whereas slowly progressing or arrested caries lesions are usually darker. ${ }^{63}$

\section{Erosion}

Tooth erosion is defined as the loss of dental hard tissue by nonbacterial chemical mechanical processes, resulting in loss of superficial enamel and visible by yellow tooth coloration. Recent lifestyle changes and the increased consumption of acidic foods and beverages have led to an increase in the prevalence of dental erosion worldwide. High prevalence of tooth erosion in 5-year-old children (from 30 to 98\%) has been reported. ${ }^{64}$ Due to lower mineralization levels and thinner primary teeth enamel compared with permanent teeth, the process of erosion is expected to be faster in children. A comparative study evaluated 60 primary and permanent teeth focusing on the erosive potential due to different type of beverages consumed and showed that surface microhardness was lower for primary teeth than for permanent teeth. ${ }^{65}$ Darker color can occur due to prolonged exposure of the underlying dentin as the enamel thins. $^{14}$

\section{Extrinsic Discoloration}

Extrinsic discoloration is outside the tooth substance and lies on the tooth surface or in the acquired pellicle. ${ }^{66}$

\section{Stains}

Black stains affect 2.4 to $16 \%$ of primary teeth. ${ }^{67}$ It starts as early as age 2 and can significantly affect the parents and have an influence on the child's personality as well as the child's self-confidence. It is a form of dental plaque characterized by high calcium and phosphate levels in addition to insoluble iron salts. ${ }^{68}$ The black stain is ferric sulfide from the interaction of hydrogen sulfide arises with gingival fluids or saliva. Another etiological factor reported is the association with some bacterial strains like Lactobacillus and Actinomyces. ${ }^{69}$ The strength of the adhesion is still under investigation; however, attractive forces, including hydrophobic 
interaction, electrostatic, and hydrogen bonds, have been reported. ${ }^{68}$ Different criteria have been proposed to establish a diagnosis. Koch et al considered the presence of dark dots, linear discoloration, and changes to the smooth surfaces of two or more different teeth. ${ }^{70}$ On the other hand, Koch et al relied on the presence or absence of the dental plaque as follows: (1) no line, (2) incomplete coalescence of pigmented dots, or (3) continuous line formed by pigmented spots. ${ }^{70}$

It is essential to state that there is a relationship between the prevalence of dental caries and the presence of black stain. A recent meta-analysis that included 13 studies concluded that individuals experiencing black stain had lower caries experience in comparison to those without the condition. $^{71}$

Iron deficiency affects more than two billion people in the world and is one of the most common nutritional deficiencies. Iron can be found in dental hard tissue, including enamel and dentin. ${ }^{72}$ The most common cause of iron deficiency is the insufficient intake of iron in the diet. This is mostly treated by food and syrup supplements that contain iron salts. ${ }^{73}$ Black stain is a type of complication that results from oral iron supplements to help children fight anemia. ${ }^{74}$

Green stain is a type of stain of unknown etiology that affects more male children than female. However, it has been reported that it is an action of chromogenic bacteria. The most common teeth affected are the maxillary central incisor, especially on the gingival third labially. Mouth breathing is also considered as a contributing factor. ${ }^{27}$

The orange stain is less frequent when compared with previously mentioned black and green stains, and can be removed more easily. Types of chromogenic bacteria, including Serratia marcescens and Flavobacterium lutescens, have been reported as potential etiology in combination with poor oral hygiene. Orange stain is mostly noted on the gingival third of the tooth. ${ }^{75}$

As it is not possible to remove extrinsic stains by daily oral care, professional cleaning is usually required to meet the child aesthetic demands. Brushing with pumice powder following simple scaling is usually enough; however, the stain tends to recur. As a result, the enamel beneath the stain might be affected, resulting in initial demineralization or roughness. 27,68

\section{Stannous Fluoride Pigmentation}

Research done by Muhler in 1946 reported the efficiency of using stannous fluoride ( $\mathrm{SnF} 2$ ) on reducing dental caries. The study compared two groups receiving 2 and $4 \%$ of SnF2, respectively, with a control group showed that children received $\mathrm{SnF} 2$ had experienced $37 \%$ reduction in new caries surface. ${ }^{76}$ Upon applying $8 \%$ SnF2 solution, certain areas of the tooth become discolored, ranging from light brown to black depending on the depth and involvement of caries. Furthermore, pigmentations affect caries and precarious enamel following stannous fluoride application. ${ }^{77}$

\section{Silver Diamine Fluoride Stain}

Silver diamine fluoride (SDF) is one of the recently recommended materials to prevent and arrest dental caries owing

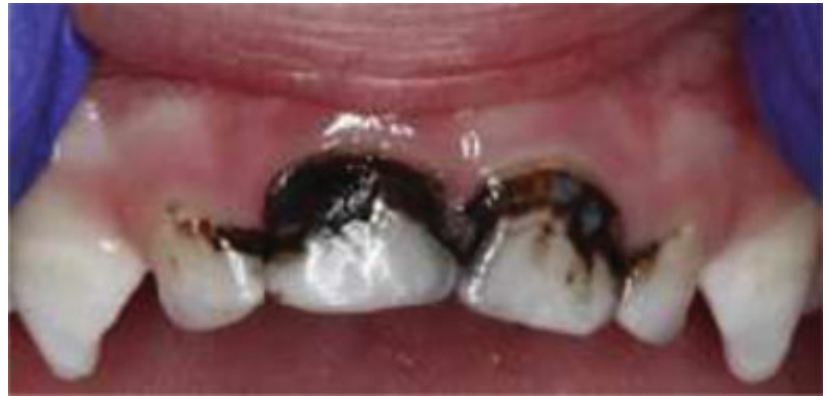

Fig. 5 Stain following silver diamine fluoride application.

to the low cost of using SDF. ${ }^{78}$ SDF is a colorless alkaline solution containing silver and fluoride, which forms a complex with ammonia. The ammonia ions combine with silver ions to produce a complex called the diamine-silver ion.

Several studies have demonstrated the efficacy use of this type of fluoride in treating carious primary dentition. ${ }^{79}$ Thus, SDF usage could increase the cost-effectiveness of health care and improve oral health, ultimately reducing the need for urgent care. ${ }^{80}$ Since the 1940 s, silver components have been developed and used for caries prevention and it has been used in Japan for more than 40 years. Moreover, in Hong Kong and China, a trial compared the effect of $38 \%$ SDF and 5\% sodium fluoride varnish and found that the SDF is more effective arresting primary teeth caries. ${ }^{78}$ Other studies have reported a caries arrest rate of $81 \%{ }^{81}$ SDF application is affected by the concentration used and the number of times applied per year, showing that $38 \%$ SDF treatment is more recommended than $12 \%$ SDF, especially when applied twice a year. ${ }^{81}$

Regarding the mechanism of action, the antimicrobial effect is a result of silver particles acting on proteins. Moreover, the black stain of carious dentin and enamel resulting from SDF silver particles is included in - Fig. 5. A recent scoping review stated that "The aesthetic perception of black staining associated with SDF is not directly related to acceptability and satisfaction of patients and parents but related to a lower professional's acceptance." 82

\section{Conclusion}

It is essential that health care professionals be familiar with the causes of primary teeth staining and pigmentation. A better knowledge will guide the clinician to a proper diagnosis and facilitate the referral to a specialized pediatric dentist that will provide proper dental care. Children's teeth change until physiological exfoliation. The ultimate aim for pediatric dentistry is the proper management of primary dentition until normal exfoliation. Several strategies can be utilized, such as using fluoride varnish for preventing caries and erosion. A restorative approach, including fillings and preformed crowns, might be considered. Bleaching can be applied on traumatized primary incisors. Sometimes, the extraction of primary teeth is the only option to treat the child's signs and symptoms.

Primary dentition is crucial due to its close relation to the developing permanent dentition. Considering healthy 
permanent dentition is a priority when planning treatment of primary teeth. Stains are multifactorial, but with proper management, the effect on the successor teeth can be limited. Recent aesthetical demands are increasing for both children and parents or caregiver, avoiding factors that contribute to teeth staining is mandatory when possible. Stained and discolored teeth have a great effect on aesthetics, which might lead to lower self-confidence. Educating children and parents on treatment management and good oral health is mandatory to improve the child's well-being for a better smile.

\section{Funding}

None.

\section{Conflict of Interest \\ None declared.}

\section{References}

1 Thomas CW, Primosch RE. Changes in incremental weight and well-being of children with rampant caries following complete dental rehabilitation. Pediatr Dent 2002;24(02):109-113

2 Anderson HK, Drummond BK, Thomson WM. Changes in aspects of children's oral-health-related quality of life following dental treatment under general anaesthesia. Int J Paediatr Dent 2004;14 (05):317-325

3 Mahmoodian J, Hashemi S. The frequency of different types of primary teeth discoloration in children in paediatric department of Faculty of Dentistry, Tehran University of Medical Sciences, in 1999-2001. Journal of Dentistry of Tehran University of Medical Sciences 2004;1(02):63-65

4 Joiner A, Luo W. Tooth colour and whiteness: a review. J Dent 2017;67S:S3-S10

5 Weerheijm KL. Molar incisor hypomineralization (MIH): clinical presentation, aetiology and management. Dent Update 2004231 (01):9-12

6 O'Brien WJ, Groh CL, Boenke KM. One-dimensional color order system for dental shade guides. Dent Mater 1989;5(06):371-374

7 Judd DB, Wyszecki G353-354 New York: Wiley 1975. Colour in Business Science and Industry. 3rd ed New York: Wiley; 1975: 353-354

8 Bridgeman I. The Nature of Light and Its Interaction with Matter. Colour Physics for Industry UK: H Charlesworth \& Co Ltd.; 1987: 1-34

9 Munsell A. A Color Notation. Baltimore, Maryland: Munsell Color Company; 1981

10 McLaren KE. Colour Space, Colour Scales and Colour Difference. Colour Physics for Industry Huddersfield: H. Charlesworth \& Co Ltd.; 1987:97-115

11 Hasegawa A, Ikeda I, Kawaguchi S. Color and translucency of in vivo natural central incisors. J Prosthet Dent 2000;83(04):418-423

12 Alman DH. Colour Physics for Industry, edited by Roderick McDonald, Society of Dyers and Colourists, Bradford. Color Res Appl 1988;13(04):264-5

13 Bergen S. Dentists Color Matching Skills. Los Angeles: University of California; 1975

14 Watts A, Addy M. Tooth discolouration and staining: a review of the literature. Br Dent J 2001;190(06):309-316

15 Kim JW, Simmer JP. Hereditary dentin defects. J Dent Res 2007;86 (05):392-399

16 Miletich I, Sharpe PT. Normal and abnormal dental development. Hum Mol Genet 2003;112 Spec No 1R69-73

17 Balic A. Concise review: cellular and molecular mechanisms regulation of tooth initiation. Stem Cells 2019;37(01):26-32
18 De Menezes Oliveira MA, Torres CP, Gomes-Silva JM, et al. Microstructure and mineral composition of dental enamel of permanent and deciduous teeth. Microsc Res Tech 2010;73 (05):572-577

19 Finn SB. Morphology of primary teeth. In: Finn SB, ed. Clinical Pedodontics. 3rd edition. Philadelphia: Wiley Blackwell; 1967

20 Ash MM, Nelson SJ. Wheeler's dental anatomy. Physiology and Occlusion 2003;8:15

21 Cavalheiro CP, Souza PS, Pedrotti D. , et al. Shortening of etching time of the dentin in primary teeth restorations: a randomized clinical trial. Braz Oral Res 2020; $\cdots: 34$

22 Kalkani M, Ashley P. The role of paediatricians in oral health of preschool children in the United Kingdom: a national survey of paediatric postgraduate specialty trainees. Eur Arch Paediatr Dent 2013;14(05):319-324

23 Dickson-Swift V, Kenny A, Gussy M, McCarthy C, Bracksley-O'Grady $\mathrm{S}$. The knowledge and practice of pediatricians in children's oral health: a scoping review. BMC Oral Health 20202520(01):211

24 Alazmah AS. Involving Children In Oral Health Research: Are We Doing Enough? (Doctoral dissertation, UCL (University College London))

25 Kaur P, Singh S, Mathur A, et al. Impact of dental disorders and its influence on self-esteem levels among adolescents. . J Clin Diagn Res 2017;11(04):ZC05-ZC08

26 Marshman Z, Rodd HD. The psychosocial impacts of developmental enamel defects in children and young people. In: Planning and Care for Children and Adolescents with Dental Enamel Defects Berlin: Springer; 2015:85-97

27 Hattab FN, Qudeimat MA, al-Rimawi HS. Dental discoloration: an overview. J Esthet Dent 1999;11(06):291-310

28 Manuel ST, Abhishek P, Kundabala M. Etiology of tooth discoloration-a review. 2010;18(02):56-63

29 Duggal M, Nazzal H. Restorative management of dental enamel defects in the primary dentition. In: Planning and Care for Children and Adolescents with Dental Enamel Defects Berlin: Springer; 2015:123-137

30 Hattab FN, Wei SH. Dietary sources of fluoride for infants and children in Hong Kong. Pediatr Dent 1988;10(01):13-18

31 Burt BA. The changing patterns of systemic fluoride intake. J Dent Res 1992;71(05):1228-1237

32 Hattab FN, el-Daher N, Salem NS. Fluoride content of drinkingwater and beverages in Jordan. World Health Forum 1997;18(01): 69-70

33 DenBesten PK. Biological mechanisms of dental fluorosis relevant to the use of fluoride supplements. Community Dent Oral Epidemiol 1999;27(01):41-47

34 Fejerskov O, Ekstrand J, Burt BA, Eds. Fluoride in dentistry. Munksgaard; 1996

35 Feinman RA, Goldstein RE, Garber DA. Bleaching teeth. Quintessence Publ Co1987

36 Mello HS. The mechanism of tetracycline staining in primary and permanent teeth. ASDC J Dent Child 1967;34(06):478-487

37 Urist MR, Ibsen KH. Chemical reactivity of mineralized tissue with oxytetracycline. Arch Pathol 1963;76:484-496

38 Steward DJ. The re-incorporation in calcified tissues of tetracycline released following its deposition in the bone of rats. Arch Oral Biol 1973;18(06):759-764

39 Cheek CC, Heymann HO. Dental and oral discolorations associated with minocycline and other tetracycline analogs. J Esthet Dent 1999;11(01):43-48

40 McEvoy SA. Chemical agents for removing intrinsic stains from vital teeth. II. Current techniques and their clinical application. Quintessence Int 1989;20(06):379-384

41 Pöyhönen H, Nurmi M, Peltola V, Alaluusua S, Ruuskanen O, Lähdesmäki T. Dental staining after doxycycline use in children. J Antimicrob Chemother 2017;72(10):2887-2890

42 Gadhia K, McDonald S, Arkutu N, Malik K. Amelogenesis imperfecta: an introduction. Br Dent J 2012;212(08):377-379 
43 Aldred MJ, Savarirayan R, Crawford PJ. Amelogenesis imperfecta: a classification and catalogue for the 21st century. Oral Dis 2003;9 (01):19-23

44 Fearne JM, Bryan EM, Elliman AM, Brook AH, Williams DM. Enamel defects in the primary dentition of children born weighing less than 2000 g. Br Dent J 1990;168(11):433-437

45 Negre-Barber A, Montiel-Company JM, Boronat-Catalá M, CataláPizarro M, Almerich-Silla JM. Hypomineralized second primary molars as predictor of molar incisor hypomineralization. Sci Rep 2016;6(01):31929

46 Velló MA, Martínez-Costa C, Catalá M, Fons J, Brines J, GuijarroMartínez R. Prenatal and neonatal risk factors for the development of enamel defects in low birth weight children. Oral Dis 2010;16(03):257-262

47 Takaoka LA, Goulart AL, Kopelman BI, Weiler RM. Enamel defects in the complete primary dentition of children born at term and preterm. Pediatr Dent 2011;33(02):171-176

48 Barron MJ, McDonnell ST, Mackie I, Dixon MJ. Hereditary dentine disorders: dentinogenesis imperfecta and dentine dysplasia. Orphanet J Rare Dis 2008;3(01):31

49 Shields ED, Bixler D, el-Kafrawy AM. A proposed classification for heritable human dentine defects with a description of a new entity. Arch Oral Biol 1973;18(04):543-553

50 Sarapultseva M, Leleko A, Sarapultsev A. Case report: Rehabilitation of a child with dentinogenesis imperfecta with CAD/CAM approach: three-year follow-up. Spec Care Dentist 2020

51 Day PF, Flores MT, O'Connell AC, et al. International Association of Dental Traumatology guidelines for the management of traumatic dental injuries: 3 . Injuries in the primary dentition. Dent Traumatol 2020;36(04):343-359

52 de Blanco LP. Treatment of crown fractures with pulp exposure. Oral Surg Oral Med Oral Pathol Oral Radiol Endod. 1996;82(05): 564-568

53 Rocha MJ, Cardoso M. Federal University of Santa Catarina endodontic treatment of traumatized primary teeth - part 2. Dent Traumatol 2004;20(06):314-326

54 Holan G, Fuks AB. The diagnostic value of coronal dark-gray discoloration in primary teeth following traumatic injuries. Pediatr Dent 1996;18(03):224-227

55 Goldstein RE. Bleaching teeth: new materials-new role. J Am Dent Assoc 1987; 1(Spec No):44E-52E

56 Aguiló L, Gandía JL. Transient red discoloration: report of case. ASDC J Dent Child 1998;65(05):346-348, 356

57 Wilson CF. Management of trauma to primary and developing teeth. Dent Clin North Am 1995;39(01):133-167

58 Mass E, Hassan A, Zilberman U. Long-term in-vivo effect of various restorative materials on enamel and dentin of primary molars. Quintessence Int 2017;48(08):633-638

59 Ortiz-Ruiz AJ, Pérez-Guzmán N, Rubio-Aparicio M, Sánchez-Meca J. Success rate of proximal tooth-coloured direct restorations in primary teeth at 24 months: a meta-analysis. Sci Rep 2020;10 (01):6409

60 Andreasen FM, Sewerin I, Mandel U, Andreasen JO. Radiographic assessment of simulated root resorption cavities. Endod Dent Traumatol 1987;3(01):21-27

61 Hon L, Mohamed A, Lynch E. Reliability of colour and hardness clinical examinations in detecting dentine caries severity: a systematic review and meta-analysis. Sci Rep 2019;9(01):6533

62 Kidd EA, Joyston-Bechal S, Smith MM. Staining of residual caries under freshly-packed amalgam restorations exposed to tea/chlorhexidine in vitro. Int Dent J 1990;40(04):219-224

63 Ghaderi F, Jowkar Z, Tadayon A. Caries color, extent, and preoperative pain as predictors of pulp status in primary teeth. Clin Cosmet Investig Dent 2020;12:263-269
64 Tvilde BN, Virtanen JI, Bletsa A, Graue AM, Skaare AB, Skeie MS. Dental erosive wear in primary teeth among five-year-olds Bergen, Norway. [published online ahead of print]Acta Odontol Scand 2020:1-7

65 Lussi A, Kohler N, Zero D, Schaffner M, Megert B. A comparison of the erosive potential of different beverages in primary and permanent teeth using an in vitro model. Eur J Oral Sci 2000; 108(02):110-114

66 Manuel ST, Abhishek P, Kundabala M. Etiology of tooth discoloration-a review. Manipal Academy of Higher Education 2010;18 (02):56-63

67 Boka V, Trikaliotis A, Kotsanos N, Karagiannis V. Dental caries and oral health-related factors in a sample of Greek preschool children. Eur Arch Paediatr Dent 2013;14(06):363-368

68 Rachid F, Mehdi HE. Black stains in primary teeth: overview. Pediatr Dent Care 2016;1(123):2

69 Stenudd C, Nordlund A, Ryberg M, Johansson I, Källestål C, Strömberg N. The association of bacterial adhesion with dental caries. J Dent Res 2001;80(11):2005-2010

70 Koch MJ, Bove M, Schroff J, Perlea P, García-Godoy F, Staehle HJ. Black stain and dental caries in schoolchildren in Potenza, Italy. ASDC J Dent Child 2001;68(5-6):353-355, 302

71 de Rezende VS, Fonseca-Silva T, Drumond CL, Ramos-Jorge ML, Paiva SM, Vieira-Andrade RG. Do patients with extrinsic black tooth stains have a lower dental caries experience? A systematic review and meta-analysis. Caries Res 2019;53(06):617-627

72 De-Regil LM, Jefferds ME, Sylvetsky AC, Dowswell T. Intermittent iron supplementation for improving nutrition and development in children under 12 years of age. Cochrane Database Syst Rev 2011;2011(12):CD009085

73 Adcock KG, Hogan SM. Extrinsic iron staining in infant teeth from iron-fortified formula and rice cereal. J Pediatr Pharmacol Ther 2008;13(03):162-165

74 Pani SC, Alenazi FM, Alotain AM, Alanazi HD, Alasmari AS. Extrinsic tooth staining potential of high dose and sustained release iron syrups on primary teeth. BMC Oral Health 2015;15 (01):90

75 Carranza FA, Newman M. Clinical Periodontology. New York: Elsevier; 1996

76 Muhler JC. Stannous fluoride. Preventive Dentistry Syllabus 1962: 288

77 Wellock WD, Maitland A, Brudevold F. Caries increments, tooth discoloration, and state of oral hygiene in children given single annual applications of acid phosphate-fluoride and stannous fluoride. Arch Oral Biol 1965;10(03):453-460

78 Duangthip D, Chen KJ, Gao SS, Lo ECM, Chu CH. Managing early childhood caries with atraumatic restorative treatment and topical silver and fluoride agents. Int J Environ Res Public Health 2017; 14(10):1204

79 Chibinski AC, Wambier LM, Feltrin J, Loguercio AD, Wambier DS, Reis A. Silver diamine fluoride has efficacy in controlling caries progression in primary teeth: a systematic review and metaanalysis. Caries Res 2017;51(05):527-541

80 Craig GG, Powell KR, Price CA. Clinical evaluation of a modified silver fluoride application technique designed to facilitate lesion assessment in outreach programs. BMC Oral Health 2013;13(01): 73

81 Fung MHT, Duangthip D, Wong MCM, Lo ECM, Chu CH. Arresting dentine caries with different concentration and periodicity of silver diamine fluoride. JDR Clin Trans Res 2016;1(02):143-152

82 Magno MB, Silva LPD, Ferreira DM, Barja-Fidalgo F, FonsecaGonçalves A. Aesthetic perception, acceptability and satisfaction in the treatment of caries lesions with silver diamine fluoride: a scoping review. Int J Paediatr Dent 2019;29(03):257-266 